\title{
Nude Mouse
}

National Cancer Institute

\section{Source}

National Cancer Institute. Nude Mouse. NCI Thesaurus. Code C14239.

A hairless mutant mouse with thymic hypoplasia, lacking T-cells. They are unable to reject transplants. 